Correspondence

Pinaki Panigrahi

ppanigrahi@unmc.edu

Received 18 October 2010

Accepted 16 December 2010

\section{Extended-spectrum $\beta$-lactamase-producing Gram-negative bacteria causing neonatal sepsis in India in rural and urban settings}

\author{
Dinesh S. Chandel, ${ }^{1,2}$ Judith A. Johnson, ${ }^{3}$ Rama Chaudhry, ${ }^{4}$ \\ Nidhi Sharma, ${ }^{5}$ Nandita Shinkre, ${ }^{5}$ Sailajanandan Parida, ${ }^{6}$ Pravas R. Misra ${ }^{2}$ \\ and Pinaki Panigrahi ${ }^{7}$
}
${ }^{1}$ Department of Environmental, Agricultural and Occupational Health, Center for Global Health and Development, College of Public Health-University of Nebraska Medical Center, Omaha, NE, USA
${ }^{2}$ Asian Institute of Public Health, Bhubaneswar, Orissa, India
${ }^{3}$ Emerging Pathogens Institute, University of Florida, Gainesville, FL, USA
${ }^{4}$ Department of Microbiology, All India Institute of Medical Sciences, New Delhi, India
${ }^{5}$ BYL Nair Hospital and TN Medical College, Mumbai, India
${ }^{6}$ SCB Medical College, Orissa, India
${ }^{7}$ Departments of Epidemiology and Pediatrics, Center for Global Health and Development, College of Public Health-University of Nebraska Medical Center, Omaha, NE, USA

\begin{abstract}
Extended-spectrum $\beta$-lactamase (ESBL)-producing Gram-negative bacilli (GNB) are of increasing clinical concern in all age groups worldwide. Whilst sepsis continues to be the leading cause of morbidity and mortality in Indian neonates in the community, identification of microbiological attributes in this population is lacking. This population-based study enrolled 1738 infants with a diagnosis of clinical sepsis at four participating centres in India. Each study site conducted Bactec blood culture, identified bacterial species by API test and stored isolates at $-70{ }^{\circ} \mathrm{C}$. From $252 \mathrm{GNB}$ isolates, 155 (113 Klebsiella species, 21 Escherichia coli and 21 other) were subjected to drug susceptibility testing, ESBL phenotyping and testing for clonal relatedness of ESBL strains by PFGE. The results demonstrated that Klebsiella species and E. coli are the most common GNB causes of neonatal sepsis in India, and over one-third are ESBL producers in both community and hospital settings. ESBL-producing strains exhibited frequent co-resistance to aminoglycosides and ciprofloxacin, but remained susceptible to imipenem. PFGE analysis revealed extensive genetic diversity within the ESBL-producing isolates, showing multiple profiles (total of 23 ). Over $40 \%$ of all ESBL-producing isolates formed three pulsed-field profiles (PFP I-III), with PFP-II being the largest cluster ( $>20 \%$ of all ESBL-producing isolates), sharing strains from two distant locations.

Identification of a common clone at two geographically distant centres indicated that predominant clones with increased virulence may exist, even in the absence of any clear outbreak. The presence of ESBL-producing strains in community infants with no prior history of hospitalization or antibiotic use dictates heightened vigilance and further studies on the ecology of these organisms.
\end{abstract}

\section{INTRODUCTION}

Neonatal sepsis is the most common primary diagnosis for admission of infants to Indian hospitals and has long been implicated in the bulk ( $52 \%$ ) of newborn deaths in villages

Abbreviations: AMC, amoxicillin/clavulanate; AMK, amikacin; AMP, ampicillin; BBS, Capital Hospital, Bhubaneshwar; CAZ, ceftazidime; CFP, cefoperazone; CIP, ciprofloxacin; CTX, cefotaxime; ESBL, extended-spectrum $\beta$-lactamase; GEN, gentamicin; GNB, Gram-negative bacilli; IPM, imipenem; IR, inhibitor resistant; MUM, BYL Nair Hospital, Mumbai; PFP, pulse-field profile; RKL, Ispat General Hospital, Rourkela.
(National Neonatal-Perinatal Database, 1997). More recent studies ascribe about $40 \%$ of neonatal deaths to sepsis in the community (National Neonatology Forum NNPD Network, 2005). The incidence of neonatal sepsis among hospitalborn infants is relatively higher in infants of low birth weight (Lautenbach et al., 2001), another major cause of morbidity and hospital admission in India. Descriptions of the microbiology of neonatal infections are limited to hospital studies, and higher mortality has been reported with infection due to extended-spectrum $\beta$-lactamase (ESBL)producing Gram-negative bacilli (GNB) in this setting (Jain 
et al., 2003). The increased mortality is generally considered not to be due to increased disease severity in patients presenting with ESBL-positive infections, but rather due to higher rates of treatment failure (Kim et al., 2002). Recent studies, however, implicate increased cell invasion and expression of fimbrial adhesins with ESBL production (Sahly et al., 2008). Whilst ESBL-producing GNB continue to be important nosocomial pathogens in Western countries, as well as in developing countries such as in India (Anandan et al., 2009), there is a paucity of information on their prevalence in community-acquired infections. Very recent data from France have begun to suggest against conventional wisdom and indicate that some ESBL infections in neonates may indeed be community-acquired via mother-to-neonate transmission (Dubois et al., 2010).

ESBL-producing isolates, in addition to being resistant to $\beta$-lactam antibiotics including third-generation cephalosporins, often exhibit resistance to other classes of drugs such as aminoglycosides, cotrimoxazole, tetracycline and fluoroquinolones. Thus, they pose a formidable challenge with limited therapeutic options, particularly in resourcechallenged countries (Decré et al., 1998; Bagattini et al., 2006). In rural India, the majority of births still take place in the home environment and the bulk of the babies do not receive hospital care. Village-level practitioners with no formal medical training often administer empiric antibiotics available on the market without a prescription. No information is currently available on microbiological resistance in the community and how it compares with that seen in hospitals. Consequently, rural clinics and small hospitals do not follow any specific policy for antibiotic use in specific medical conditions.

The present study included identification and genotyping, by PFGE, of ESBL-positive Klebsiella species and Escherichia coli isolates from confirmed neonatal sepsis in hospital- and community-acquired infections from different participating centres in India. ESBL infections were also compared among hospitals and in the community-born infant population. To the best of our knowledge, this is the first multicentre and geographically diverse molecular epidemiological survey of ESBL-producing GNB implicated in neonatal sepsis in a developing country setting.

\section{METHODS}

Study sites and patient population. This study involved four different centres in India. Babies were enrolled at BYL Nair Hospital, Mumbai (MUM), Capital Hospital, Bhubaneshwar (BBS), and Ispat General Hospital, Rourkela (RKL). The All India Institute of Medical Sciences, New Delhi, served as the microbiology reference laboratory and performed the molecular analyses. Mumbai is a major metropolitan area in western India and MUM serves a predominantly urban population under the municipal system providing free medical services. Babies enrolled at this site were all born in hospital, and the mothers came from the urban and slum neighbourhoods. BBS and RKL are in Orissa state, located in the relatively resource-poor eastern region, recording one of the highest neonatal and infant mortality rates in India. Bhubaneshwar is the capital city of Orissa; however, a significant proportion of babies enrolled at Capital Hospital (a government-run hospital) were primarily from neighbouring rural villages of the Khurda district. Ispat General Hospital is in a steel township in western Orissa, where the bulk of enrolled infants came from neighbouring tribal villages in the district of Sundargarh. Infants enrolled at these two sites included those born at home in the adjacent villages and at the same hospitals where mothers came from the neighbouring rural area. All infants ( $<60$ days old) born in the community or at the hospitals with a primary diagnosis of clinical sepsis, based on the World Health Organization-specified criteria, were enrolled in the study (WHO Young Infants Study Group - Weber et al., 2003). Exclusion criteria included major congenital anomalies and refusal of informed consent by parents for blood draws. Clinical sepsis also included culture-negative patients as well as those presenting with respiratory symptoms (pneumonia). The study included cases of sepsis in home-born as well as hospital-born infant populations. Single blood samples were obtained from each infant for microbiological culture identification and drug-susceptibility testing prior to initiation of antimicrobial therapy. Trained clinical staff at the study centres collected blood using strict sterile techniques. The study was approved by local (state) ethical review committees and Institutional Review Boards at participating centres in India and the USA. Written informed consent was taken from one parent. Over the 3year study period (2002-2005), a total of 2810 infants were screened for signs and symptoms of sepsis and 1738 were enrolled in the study.

Bacterial isolates. A blood sample was collected for culture from each infant immediately after enrolment and prior to initiation of treatment. All samples were inoculated into BACTEC PEDS PLUS/F medium and processed using the Bactec 9050 System (Becton Dickinson). Positive bottles were subcultured on blood agar, chocolate agar and MacConkey agar plates. Standard biochemical tests were performed along with the use of API test strips (bioMérieux) for identification. Bacterial isolates were stored frozen in L-broth with $20 \%$ glycerol at $-70{ }^{\circ} \mathrm{C}$. Blood culture plates showing growth of Corynebacterium, Propionibacterium, diphtheroids, Micrococcus and Bacillus species were considered contaminants and therefore not included in the culture positivity. From a total of 252 GNB isolates, 155 (113 Klebsiella species, 21 E. coli and 21 other GNB) strains were included in this study. These strains widely represented our Orissa state community $(n=31)$ and linked township hospitals $(n=45)$. Gram-negative isolates $(n=79)$ from a larger tertiarycare hospital at Mumbai were also studied for comparison.

Drug susceptibility and ESBL detection. Strain identification and standard susceptibility testing were carried out by the respective centres. Primarily, all blood isolates were subjected to routine bacterial culture identification and drug-susceptibility testing. Based on our preliminary drug-susceptibility data, strains showing unusually high resistance to one or more third-generation cephalosporins were identified as potential bearers of ESBL enzyme activity. Moreover, increased rates of treatment failure due to this group of drugs prompted us to investigate the ESBL trends in the community infants who had no prior history of hospital exposure. For the purpose of this study, strains were shipped to the reference laboratory where identifications were confirmed and strains were tested against a panel of antibiotics. Antibiotic susceptibility testing was carried out by a disc-diffusion technique using National Committee for Clinical Laboratory Standards guidelines (NCCLS, 2001). The $\beta$-lactam antibiotics tested were ampicillin (AMP), amoxicillin/clavulanate (AMC), cefoperazone (CFP), cefotaxime (CTX), ceftazidime (CAZ) and imipenem (IPM). Amikacin (AMK), ciprofloxacin (CIP) and gentamicin (GEN) were also tested. Confirmation of ESBL activity in E. coli and Klebsiella was undertaken by disc synergy testing of CTX and CAZ in the presence and absence of clavulanic acid (Jarlier et al., 1988; NCCLS, 2001). Standard strains of K. pneumoniae (ATCC 700603) and E. coli (ATCC 25922) were used as positive and negative controls, respectively. All Gram-negative isolates were tested against the above panel of antibiotics. Strains exhibiting co-resistance to at 
least one third-generation cephalosporin (CFP/CTX/CAZ), a $\beta$ lactam (AMP), an aminoglycoside (GEN or AMK) and a fluoroquinolone (CIP) were considered multidrug-resistant pathogens.

Genotyping of bacterial isolates. DNA typing was performed by PFGE, a widely accepted standard tool in genotyping of bacterial pathogens (Struelens, 1996). Briefly, a $200 \mu \mathrm{l}$ cell suspension of an overnight bacterial culture was mixed with an equal volume of $1.6 \%$ low-melting-point agarose/SDS mix (held at $55{ }^{\circ} \mathrm{C}$; Sigma). Gel plugs were poured using plug moulds, allowed to solidify at room temperature and treated individually with lysis buffer containing proteinase $\mathrm{K}$. The plugs were finally washed in plug wash buffer (three times) at $50{ }^{\circ} \mathrm{C}$ and stored until restriction analysis. Cut portions comprising one-third of each DNA plug were digested with $\mathrm{XbaI}$ enzyme overnight at $25{ }^{\circ} \mathrm{C}$. The macro-restricted DNA plugs were resolved in a $1 \%$ Seakem GTG/agarose gel (FMC BioProducts) at $6 \mathrm{~V}$ $\mathrm{cm}^{-1}$ with a $120^{\circ}$ angle, pulsed at 5-45 s. The gels were electrophoresed for $20 \mathrm{~h}$ at $14{ }^{\circ} \mathrm{C}$ in a CHEF-DR II apparatus (Bio-Rad). Digitized images from stained gels were subjected to cluster mapping by GelCompar II-based similarity coefficient analysis (Applied Maths) and analysed in accordance with the published consensus on interpretation of PFGE data (Tenover et al. 1995; Struelens, 1996).

Statistical analysis. We performed $\chi^{2}$ analyses to compare differences in ESBL positivity between smaller hospitals and the community in Orissa state and between small township hospitals and the larger tertiary-care centre at Mumbai. All analyses were performed using SAS version 9.1.9 and the results were considered significant when $P<0.05$.

\section{RESULTS}

Blood culture revealed an overall culture positivity of $42 \%$ (281/668), $17.5 \%(106 / 606)$ and $19.6 \%$ (91/464) in infants at the MUM, RKL and BBS sites, respectively. GNB isolates $(n=252)$ comprised the majority $(53 \%)$ of all culturepositive cases $(n=478)$, followed by Gram-positive $(33 \%)$ and fungal $(6 \%)$ isolates. A significant number of sepsis cases $(8 \%)$ was also attributed to mixed infections (Table 1). A total of 113 Klebsiella species and $21 \mathrm{E}$. coli were available (recovered from frozen stocks) for ESBL testing and molecular subtyping at the All India Institute of Medical Sciences reference laboratory.
Most of these isolates showed widespread resistance to multiple drugs. High-level resistance to AMP was observed in $98 \%$ of Klebsiella and $81 \%$ of E. coli cases, whilst other GNB showed complete resistance to this drug. Klebsiella and E. coli also exhibited increased resistance to both AMC and GEN (50\%), as well as to CIP (35\%). Among the other GNB, Enterobacter species showed alarmingly high resistance $(>95 \%)$ to the ESBL inhibitor AMC. However, all isolates were susceptible to IPM, with more than $70 \%$ showing co-sensitivity to AMK (Table 2).

More than $50 \%$ of E. coli (11/21) and $46 \%$ of Klebsiella species (52/113) were resistant to one or more thirdgeneration cephalosporins, most showing complete resistance to all three. Other GNB (mostly Enterobacter species) also recorded high-level resistance to third-generation cephalosporins (75\%). Double disc diffusion confirmed that approximately one-third of Klebsiella species (35/113) and E. coli (7/21) received from all sites were ESBL producers (Table 3 ). The percentage of ESBLs among Klebsiella and E. coli was highest in MUM (35\%), followed by RKL (33\%) and BBS (13\%). In addition, three isolates each of Klebsiella and E. coli were also inhibitor resistant (IR), suggesting that another class of $\beta$-lactamase may be responsible for the resistance. All ESBL producers showed more frequent co-resistance to multiple drugs.

\section{ESBL antibiotypes}

Based on antibiotic susceptibility profiling, overall 15 antibiotypes (Ab1-Ab15) for 42 isolates were assigned. Of these, the predominant type, Ab1 $(n=17)$, was susceptible only to IPM. Type Ab2 $(n=12)$ displayed co-sensitivity to AMK and IPM and included $50 \%$ of the IR strains. However, type Ab15 (a single isolate) was sensitive to the largest number of antibiotics (six), including IPM. The remaining ESBL isolates $(n=12)$ displayed individually unique antibiotype patterns: Ab3-Ab14. Only three isolates showed in vitro sensitivity to five or more antibiotics of the test panel. Table 4 shows the antibiotype distribution over

Table 1. Screening, enrolment and blood-culture positivity by site

Gram-negative pathogens, mainly Klebsiella species $(\mathrm{Kb})$ and E. coli $(\mathrm{Ec})$, were described in the present study. Mixed infections included infants with more than one pathogen on a single blood culture. From the 'other' GNB, 21 isolates (18 Enterobacter species and one each of Acinetobacter species, Citrobacter species and Bacillus species) were also tested for drug susceptibility comparison. $\mathrm{BC}+$, Blood culture positive.

\begin{tabular}{|c|c|c|c|c|c|c|c|c|c|c|}
\hline \multirow{2}{*}{$\begin{array}{l}\text { Study } \\
\text { site }\end{array}$} & \multirow{2}{*}{$\begin{array}{c}\text { No. } \\
\text { screened }\end{array}$} & \multirow{2}{*}{$\begin{array}{c}\text { No. } \\
\text { enrolled }\end{array}$} & \multirow[t]{2}{*}{$\mathrm{BC}+$} & \multicolumn{4}{|c|}{ Gram-negative bacteria } & \multirow{2}{*}{$\begin{array}{c}\text { Gram-positive } \\
\text { bacteria }\end{array}$} & \multirow[t]{2}{*}{ Fungi } & \multirow[t]{2}{*}{ Mixed $^{*}$} \\
\hline & & & & Total & Ec & $\mathbf{K b}$ & Other & & & \\
\hline MUM & 837 & 668 & 281 & 180 & 15 & 77 & 88 & 49 & 27 & 25 \\
\hline RKL & 942 & 606 & 106 & 31 & 1 & 20 & 10 & 74 & 1 & 0 \\
\hline BBS & 1031 & 464 & 91 & 41 & 13 & 26 & 2 & 37 & 0 & 13 \\
\hline Total & 2810 & 1738 & 478 & 252 & 29 & 123 & 100 & 160 & 28 & 38 \\
\hline
\end{tabular}

${ }^{*}$ At BBS, 12 and three additional Klebsiella species and E. coli, respectively, were tested from the mixed pool $(n=38)$. 
Table 2. Drug-resistance pattern of the GNB isolates

A '-' indicates that all strains in the group are sensitive. The numbers represent the number of isolates resistant to the respective antibiotics; percentages are given in parentheses.

\begin{tabular}{|c|c|c|c|c|c|c|c|c|c|c|}
\hline Study site & $n$ & AMK & AMC & AMP & CFP & CTX & CAZ & CIP & GEN & IPM \\
\hline \multicolumn{11}{|l|}{ E. coli } \\
\hline BBS & 16 & - & 7 & 12 & 6 & 6 & 5 & 7 & 3 & - \\
\hline RKL & $-{ }^{*}$ & - & - & - & - & - & - & - & - & - \\
\hline Total (\%) & 21 & $2(10)$ & $11(52)$ & $17(81)$ & $10(48)$ & $11(52)$ & $9(43)$ & $9(43)$ & $7(33)$ & $-(0.0)$ \\
\hline \multicolumn{11}{|l|}{ Klebsiella } \\
\hline MUM & 56 & 18 & 38 & 56 & 38 & 37 & 39 & 33 & 45 & - \\
\hline RKL & 19 & 12 & 9 & 19 & 7 & 7 & 11 & 7 & 11 & - \\
\hline Total (\%) & 113 & $31(27)$ & $51(45)$ & $111(98)$ & $48(42)$ & $47(42)$ & $52(46)$ & $41(36)$ & $56(50)$ & $-(0.0)$ \\
\hline \multicolumn{11}{|l|}{ Other GNB } \\
\hline BBS & 1 & - & 1 & 1 & 1 & 1 & 1 & 1 & - & - \\
\hline MUM & 18 & 6 & 18 & 8 & 8 & 7 & 14 & 3 & 15 & - \\
\hline
\end{tabular}

*The RKL site had a single E. coli isolate but this was unavailable for ESBL testing at the reference centre.

multiple, yet fewer dominant, genotypes, designated pulsefield profiles (PFPs).

\section{PFGE analysis}

PFGE analysis showed extensive genetic diversity, with a total of 23 PFPs generated from 42 isolates. However, over $40 \%$ of Klebsiella isolates could be grouped into three main clusters (PFP-I to PFP-III). Type PFP-I persisted for more than 6 months, observed exclusively at MUM where the highest number $(n=28)$ of ESBL isolates were recovered. PFP-II was the largest cluster $(n=9)$ with six isolates recovered at RKL; however, three cases from MUM also shared this unique pattern. The remaining ESBL isolates did not reveal any obvious genetic resemblance and dispersed into distant clusters (PFP-IV to PFP-XXIII) with fewer isolates and singletons (Fig. 1). ESBL-positive E. coli from BBS (5/7) had distinct PFPs with heterogeneous antibiotic resistance patterns. Molecular fingerprinting helped identify predominant clones (PFP-I and PFP-II) that persisted over an extended period, reported from distant sites. Although ESBL clusters did not correlate with any outbreaks at these sites, circulation of the PFP-II clone at MUM could not be ruled out. PFGE identification of a common clone at two geographically distant centres (RKL and MUM) indicated that predominant clones with increased virulence may exist even in the absence of any clear outbreak. Careful examination of case records (place of delivery, person conducting delivery, etc.) for infants in the community revealed that infants carrying the PFP-II

Table 3. Drug-resistance trends in hospitals and in the community

The numbers represent the number of isolates resistant to the respective antibiotics; percentages are given in parentheses.

\begin{tabular}{|c|c|c|c|c|c|c|c|c|c|}
\hline Study site & Home/hospital born & $n^{*}$ & $\begin{array}{c}\text { Third- } \\
\text { generation } \\
\text { drug } \dagger\end{array}$ & AMK & AMC & AMP & CIP & GEN & ESBL \\
\hline \multirow{2}{*}{$\begin{array}{l}\text { Orissa state community } \\
\text { sites }\end{array}$} & Town hospital-born $(\mathrm{H})$ & 45 & $15(33)$ & $5(11)$ & $14(31)$ & $42(93)$ & $10(22)$ & $7(16)$ & $7(16)$ \\
\hline & $\mathrm{C}+\mathrm{H}$ & 76 & $23(30)$ & $14(18)$ & $22(29)$ & $70(92)$ & $17(22)$ & $14(18)$ & $14(18)$ \\
\hline
\end{tabular}

${ }^{\star}$ Overall GNB $(n=155)$ tested comprising Klebsiella species (113), E. coli (21) and other GNB (21).

$\dagger$ Isolates resistant to two or more antibiotics in this group (CFP, CTX and CAZ).

¥MUM caters for the urban population and had no community cases. 
Table 4. Antibiotypes of ESBL/IR strains $(n=42)$ and drug susceptibility profiles

\begin{tabular}{|llll|}
\hline $\begin{array}{l}\text { No. ESBL } \\
\text { strains (no. IR) }\end{array}$ & Antibiotype & Antibiotic susceptibility pattern & PFP distribution \\
\hline $17(1)$ & $\mathrm{Ab1} 1^{*}$ & IPM & I, II, IV, V, XV, VII, XXI \\
$12(3)$ & $\mathrm{Ab}{ }^{\dagger} \dagger$ & IPM, AMK & I, III, VI, IX, X, XI \\
$1(0)$ & $\mathrm{Ab3}$ & IPM, AMC & II \\
$1(1)$ & $\mathrm{Ab} 4$ & IPM, GEN & XVI \\
$1(1)$ & $\mathrm{Ab} 5$ & IPM. AMK, GEN & XVIII \\
$1(0)$ & $\mathrm{Ab} 6$ & IPM, AMK, AMC & VIII \\
$1(0)$ & $\mathrm{Ab} 7$ & IPM, AMC, CIP & XIX \\
$1(0)$ & $\mathrm{Ab} 8$ & IPM, AMK, CIP & XXIII \\
$1(0)$ & $\mathrm{Ab} 9$ & IPM, AMK, AMC, CIP & XXII \\
$1(0)$ & $\mathrm{Ab} 10$ & IPM, AMK, AMC, CAZ & VI \\
$1(0)$ & $\mathrm{Ab} 11$ & IPM, AMK, GEN, CAZ & XX \\
$1(0)$ & $\mathrm{Ab} 12$ & IPM, AMK, CAZ, CIP & XVII \\
$1(0)$ & $\mathrm{Ab} 13$ & IPM, AMK, AMC, CAZ, CIP & XIV \\
$1(0)$ & $\mathrm{Ab} 14$ & IPM, AMK, CFP, CTX, CIP & XII \\
$1(0)$ & $\mathrm{Ab} 15$ & IPM, AMK, AMC, CFP, CTX, CIP & XIII \\
& & & \\
\hline
\end{tabular}

*Type Ab1 also included most ESBL cases from the community: almost $50 \%$ of Ab1 isolates clustered as PFP-II type (bold) and caused the maximum treatment failures, being resistant to all antibiotics except IPM.

$\dagger \mathrm{Ab} 2$ was the second major group with isolates susceptible to AMK besides IPM. It was mainly distributed over two types, PFP-I and PFP-III (bold), and included $50 \%$ of the IR strains.

type were born in different hamlets (3-7 miles apart) where the traditional birth attendants conducting these deliveries were different. One infant was delivered by a family member.

\section{ESBLs in the hospital versus community}

Although predominantly of nosocomial origin, ESBLproducing strains were also detected in the community in this study. Among 76 culture-proven Gram-negative sepsis cases in Orissa state participating hospitals (BBS and RKL), 31 were in the community whereas 45 were acquired in the hospital. At these two sites, we observed similar drug resistance trends in both hospital-born and communityborn infants (Table 3 ). In the hospital, 7/45 cases $(15.5 \%$ ) were confirmed as ESBL-positive. In the community-born babies, $7 / 31$ isolates $(22.6 \%)$ were ESBL producers. Although the ESBL rate in the community was higher than that in smaller hospitals, this difference was not statistically significant. Six of the community ESBLs $(n=7)$ were cases due to late-onset sepsis (during days 4-60 of life) implying that acquisition of these strains was not from the mother but from the environment. Most of the late-onset sepsis strains were of the same antibiotype and pulsed-field cluster (Ab1/ PFP-II). The GNB isolates $(n=79)$ tested at MUM - a busy tertiary-care hospital that catered for the urban population (and thus had no community cases) - showed higher levels of ESBL positivity (35\%). Compared with the smaller hospitals (study sites in Orissa), the tertiary-care centre in the metropolitan area (Mumbai) showed significantly higher rates of ESBL infections $(P=0.02)$. Strikingly, MUM had over $75 \%$ of GNB resistant to the ESBL inhibitor AMC. Overall, a high rate of mortality was observed in infants with confirmed ESBL cases. About $33 \%$ of ESBL infections led to death of the infants in spite of available injectable antibiotics and other supportive care.

\section{DISCUSSION}

Resistance to antimicrobial agents is a burgeoning problem in Western and developing countries alike. Among a variety of drug-resistance traits, ESBL-producing GNB with resistance to newer cephalosporins have been posing a significant challenge in clinical practice. During the past decade, ESBL strains have frequently been implicated in neonatal infections in intensive care units at tertiary-care hospitals (Bradford, 2001; Jain et al., 2003; Cartelle et al., 2004; Krishna et al., 2007). Among the GNB reported, Klebsiella and $E$. coli appear to be the most common species in both hospital and community settings. These organisms have been responsible for several unrelated outbreaks in hospitals in the USA and Europe (Bradford, 2001), sustained nosocomial infections in Italy (Perilli et al., 2002) and single-clone neonatal intensive care unit (NICU) epidemics in France (Bagattini et al., 2006). In Spain, ESBL-producing, clonally unrelated $E$. coli strains have been reported from the community (Calbo et al., 2006). Another survey of ESBLs in stools of outpatients attending a hospital in Spain showed increased frequency of ESBL carriage in the community (Mirelis et al., 2003). Several reports of ESBL-producing members of the Enterobacteriaceae in non-hospitalized cases (Arpin et al., 2003; Rodríguez-Baño et al., 2004; Valverde et al., 2004; Ben-Ami et al., 2006) also imply that important reservoirs of these pathogens exist outside hospitals. 




$\begin{array}{lcc}\text { Strain-ID } & \text { Ab/IR } & \text { PFP } \\ \text { M 1821 A } & 8 & \text { XXIII } \\ \text { M 1831 U } & 9 & \text { XXII } \\ \text { M 25011 } & 1 & \text { XXI } \\ \text { B 6461 C } & 11 & \text { XX } \\ \text { M 0791 L } & 2 & \text { I } \\ \text { M 2201 C } & 2 & \text { I } \\ \text { M 2101 G } & 1 \text { (IR) } & \text { I } \\ \text { M 2661 O } & 2 \text { (IR) } & \text { I } \\ \text { M 3411 G } & 2 & \text { I } \\ \text { M 28411 } & 1 & \text { V } \\ \text { M 39711 } & 1 & \text { V } \\ \text { M 3391 M } & 10 & \text { VI } \\ \text { M 3501 I } & 2 \text { (IR) } & \text { VI } \\ \text { R 1211 X } & 1 & \text { VII } \\ \text { M 0591 T } & 6 & \text { VIII } \\ \text { M 11111 } & 1 & \text { IV } \\ \text { M 20011 } & 1 & \text { IV } \\ \text { M 32411 } & 1 & \text { IV } \\ \text { M 1851 O } & 15 & \text { XIII } \\ \text { M 32311 } & 1 & \text { II } \\ \text { M 32331 } & 1 & \text { II } \\ \text { M 32321 } & 1 & \text { II } \\ \text { R 1471 X } & 3 & \text { II } \\ \text { R 1521 X } & 1 & \text { II } \\ \text { R 1691X } & 1 & \text { II } \\ \text { R 1981 X } & 1 & \text { II } \\ \text { R 0111 X } & 1 & \text { II } \\ \text { R 0381 X } & 1 & \text { II } \\ \text { M 1901 C } & 2 & \text { III } \\ \text { M 1931 Q } & 2 & \text { III } \\ \text { M 1991 V } & 2 & \text { III } \\ \text { M 2031 B } & 2 & \text { III } \\ \text { M 26311 } & 14 & \text { XII } \\ \text { B 0961 } & 2 & \text { IX } \\ \text { B 13621 } & 2 & \text { X } \\ \text { B 1021 } & 2 \text { (IR) } & \text { XI } \\ \text { B 5051 D } & 13 & \text { XIV } \\ \text { M 41411 } & 1 & \text { XV } \\ \text { B 4571 F } & 4 \text { (IR) } & \text { XVI } \\ \text { M 0192 } & 12 & \text { XVII } \\ \text { B 2761 K } & 5 \text { (IR) } & \text { XVIII } \\ \text { M 0471 G } & 7 & \text { XIX } \\ & & \end{array}$

Fig. 1. Comparison of PFPs and antibiotypes for all ESBL-producing and IR isolates. The PFP-Il clone comprised $50 \%$ of the Ab1 strains. However, this unique clone was shared by strains from hospitals from two geographical locations (RKL and MUM). Most of the community isolates at RKL shared the PFP-II cluster, whilst PFP-I was assigned to five strains, all of MUM origin. Overall, ESBL isolates showed genetic heterogeneity: 23 PFPs were shared among 42 strains. The prefixes M, R and B represent the study sites: Mumbai, Rourkela and Bhubaneswar for the respective strain IDs.

In the developing country context, high levels of ESBLs (86\% Klebsiella species and $60 \%$ E. coli) in septicaemic neonates have been reported previously from India in NICUs (Ben-Ami et al., 2006). A recent study from south India also indicated extensive colonization of the NICU environment by ESBL-producing $K$. pneumoniae and suggested the transmission of these pathogens to neonates through healthcare workers (Krishna et al., 2007). ESBL phenotypes in E. coli $(60 \%)$, Klebsiella species (>50\%) and Salmonella species (8\%), with high-level co-resistance to aminoglycosides and fluoroquinolones, has also been reported, suggesting multiclonal dissemination in Indian hospital settings (The India Antimicrobial Resistance Study Group et al., 2002; Manchanda et al., 2006). A survey for 
faecal carriage of ESBLs conducted at a tertiary-care hospital in a metropolitan area of India (Mumbai) during 2004 demonstrated a carriage rate of over $10 \%$, indicating the presence of ESBL-producing flora in healthy adults (Rodrigues et al., 2005). However, except for a fatal case of splenic abscess and sepsis in an adult due to ESBL-positive K. pneumoniae infection (Thambu et al., 2006), ESBLs have not been described in the Indian community setting.

In the present study, as expected, the highest percentage of ESBLs was observed in Mumbai, an urban area with a very crowded hospital with maximum hospital births. These rates were also significantly higher than in the smaller township hospitals in Orissa. However, we also observed a high prevalence (more than one-third) of ESBL-producing Klebsiella and E. coli in septicaemic infants in the community setting at RKL and BBS, small hospitals caring for rural births. Although not statistically significant, it was alarming to note a higher rate of ESBL prevalence in the community $(22.5 \%)$ compared with local hospitals $(15.5 \%)$. This is particularly concerning as Klebsiella and E. coli represent the majority of GNB causing neonatal sepsis in this region. It should be noted that for all cases of sepsis tracked to be of community origin, mothers had no previous history of hospitalization or perinatal use of antibiotics. Similarly, the babies did not receive antibiotics until enrolment into our study, and only one baby had earlyonset ESBL sepsis. This implies that ESBLs are common in the community setting and have been acquired late after birth from the community. In addition, five of the seven community isolates producing ESBLs were genetically related and also showed a common drug susceptibility pattern (Ab1). However, upon tracking back the case histories of septic babies from the community including care providers at the time of delivery, we could not delineate any probable common infection source. They belonged to two distant Anganwadi centres/hamlets with almost no regular communication between the two, where babies were delivered by different traditional birth attendants. Also, ESBL isolates shown to be clonal by PFGE analysis were recovered at multiple time points, implying that this predominant clone apparently persisted over an extended period in this region. These observations indicate that a limited number of ESBL clones may be circulating in the community. Another intriguing finding in our study was the presence of the same PFP-II type K. pneumoniae isolates in geographically distant sites (MUM and RKL, more than 1000 miles apart). This indicates a wide distribution of this resistant clone in India, possibly due to increased virulence.

In 2006, the Antimicrobial Availability Task Force of the Infectious Diseases Society of America listed ESBL-producing Enterobacteriaceae (Klebsiella species and E. coli) as one of six problematic drug-resistant pathogens and suggested an urgent need for newer and more effective therapeutics (Talbot et al., 2006). Recent reports now describe ESBLs as a global public health threat (Pitout \& Laupland, 2008). Although known to be escalating mainly in the hospital setting, ESBLs have started to emerge in the community. It is not known whether they are truly acquired in the community, have spilled over from hospitals or have a multifocal source. The current report describes a widespread problem of ESBL-producing multidrug-resistant strains of Klebsiella and E. coli in both hospital and community settings in a developing country environment. The importance of this observation is highlighted by the very high mortality rate $(33 \%)$ in these patients, in spite of the availability of newer antibiotics and supportive hospital care. Whilst the high rate of ESBL positivity in a busy tertiary-care hospital was not unexpected, it was rather alarming to note the higher prevalence of ESBLs in the villages compared with smaller hospitals serving those villages. Our data suggest that the prevalence of these organisms in both hospital and community settings in developing countries is probably under-reported and may be higher than that in the developed world. The availability of antibiotics without a prescription, their widespread use by the general public often with suboptimal dosing and duration of therapy, and the occasional use of counterfeit drugs all contribute to the emergence of this problem. Whilst other risk factors in the mother and neonate (evaluation not within the scope of this work) in the developing world setting may provide additional insights into such problems, there is an urgent need for effective surveillance and institution of appropriate infection control measures. Screening of attending staff and mothers before delivery for ESBL carriage, isolation of carriers, barrier nursing protocols, judicious antibiotic use policies, curtailing the rampant use of broad-spectrum antibiotics in the village settings and hospitals, and use of alternative treatment modalities will help reduce the further spread of these resistant organisms.

\section{ACKNOWLEDGEMENTS}

This study was funded by NICHD (HD 40574) and The Bill and Melinda Gates Foundation, USA. We are thankful to the Government of Orissa, Department of Health \& Family Welfare, for making the necessary arrangements to conduct this research study. We extend our thanks to Professor N. K. Ganguly, former Director General, Indian Council of Medical Research, for expediting all required reviews and approvals and for giving his valuable time and advice throughout the study period during our multiple presentations and briefings with him. Amulya R. Nanda, former Secretary of Family Welfare, was very helpful in coordinating efforts to provide the necessary clearances at the ministry level in the Government of India. Drs Radhanath Satpathy, Lingaraj Pradhan and Mona Pote provided excellent clinical care to the babies admitted to our study hospitals.

\section{REFERENCES}

Anandan, S., Thomas, N., Veeraraghavan, B. \& Jana, A. K. (2009). Prevalence of extended-spectrum $\beta$-lactamase producing Escherichia coli and Klebsiella spp in a neonatal intensive care unit. Indian Pediatr 46, 1106-1107.

Arpin, C., Dubois, V., Coulange, L., André, C., Fischer, I., Noury, P., Grobost, F., Brochet, J. P., Jullin, J. \& other authors (2003). Extendedspectrum $\beta$-lactamase-producing Enterobacteriaceae in community and private health care centers. Antimicrob Agents Chemother 47, 35063514. 
Bagattini, M., Crivaro, V., Di Popolo, A., Gentile, F., Scarcella, A., Triassi, M., Villari, P. \& Zarrilli, R. (2006). Molecular epidemiology of extended-spectrum $\beta$-lactamase-producing Klebsiella pneumoniae in a neonatal intensive care unit. J Antimicrob Chemother 57, 979-982.

Ben-Ami, R., Schwaber, M. J., Navon-Venezia, S., Schwartz, D., Giladi, M., Chmelnitsky, I., Leavitt, A. \& Carmeli, Y. (2006). Influx of extended-spectrum $\beta$-lactamase-producing Enterobacteriaceae into the hospital. Clin Infect Dis 42, 925-934.

Bradford, P. A. (2001). Extended-spectrum $\beta$-lactamases in the 21 st century: characterization, epidemiology, and detection of this important resistance threat. Clin Microbiol Rev 14, 933-951.

Calbo, E., Romaní, V., Xercavins, M., Gómez, L., Vidal, C. G., Quintana, S., Vila, J. \& Garau, J. (2006). Risk factors for community-onset urinary tract infections due to Escherichia coli harbouring extended-spectrum $\beta$-lactamases. J Antimicrob Chemother 57, 780-783.

Cartelle, M., del Mar Tomas, M., Pertega, S., Beceiro, A., Dominguez, M. A., Velasco, D., Molina, F., Villanueva, R. \& Bou, G. (2004). Risk factors for colonization and infection in a hospital outbreak caused by a strain of Klebsiella pneumoniae with reduced susceptibility to expanded-spectrum cephalosporins. J Clin Microbiol 42, 4242-4249.

Decré, D., Gachot, B., Lucet, J. C., Arlet, G., Bergogne-Bérézin, E. \& Régnier, B. (1998). Clinical and bacteriologic epidemiology of extended-spectrum $\beta$-lactamase-producing strains of Klebsiella pneumoniae in a medical intensive care unit. Clin Infect Dis 27, 834-844.

Dubois, V., De Barbeyrac, B., Rogues, A.-M., Arpin, C., Coulange, L., Andre, C., M'zali, F., Megraud, F. \& Quentin, C. (2010). CTX-Mproducing Escherichia coli in a maternity ward: a likely community importation and evidence of mother-to-neonate transmission. J Antimicrob Chemother 65, 1368-1371.

Jain, A., Roy, I., Gupta, M. K., Kumar, M. \& Agarwal, S. K. (2003). Prevalence of extended-spectrum $\beta$-lactamase-producing Gramnegative bacteria in septicaemic neonates in a tertiary care hospital. J Med Microbiol 52, 421-425.

Jarlier, V., Nicolas, M. H., Fournier, G. \& Phillipon, A. (1988). Extended broad-spectrum $\beta$-lactamases conferring resistance to newer $\beta$-lactam agents in Enterobacteriaceae: hospital prevalence and susceptibility patterns. Rev Infect Dis 10, 867-878.

Kim, Y. K., Pai, H., Lee, H. J., Park, S. E., Choi, E. H., Kim, J., Kim, J.-H. \& Kim, E.-C. (2002). Bloodstream infections by extended-spectrum $\beta$ lactamase-producing Escherichia coli and Klebsiella pneumoniae in children: epidemiology and clinical outcome. Antimicrob Agents Chemother 46, 1481-1491.

Krishna, B. V. S., Patil, A. B. \& Chandrasekhar, M. R. (2007). Extended spectrum $\beta$ lactamase producing Klebsiella pneumoniae in neonatal intensive care unit. Indian J Pediatr 74, 627-630.

Lautenbach, E., Patel, J. B., Bilker, W. B., Edelstein, P. H. \& Fishman, N. O. (2001). Extended-spectrum $\beta$-lactamase-producing Escherichia coli and Klebsiella pneumoniae: risk factors for infection and impact of resistance on outcomes. Clin Infect Dis 32, 1162-1171.

Manchanda, V., Singh, N. P., Shamweel, A., Eideh, H. K. \& Thukral, S. S. (2006). Molecular epidemiology of clinical isolates of AmpC producing Klebsiella pneumoniae. Indian J Med Microbiol 24, 177-181.

Mirelis, B., Navarro, F., Miró, E., Mesa, R. J., Coll, P. \& Prats, G. (2003). Community transmission of extended-spectrum $\beta$-lactamase. Emerg Infect Dis 9, 1024-1025.

National Neonatal-Perinatal Database (1997). Neonatal morbidity and mortality: report of the National Neonatal-Perinatal Database. Indian Pediatr 34, 1039-1042.
National Neonatology Forum NNPD Network (2005). Report of the National Neonatal-Perinatal Database (2002-03). Available from http://www.nnfi.org/images/NNPD_2002-03.pdf

NCCLS (2001). Zone diameter interpretive standards. NCCLS global information supplement 21: 40-71.

Perilli, M., Dell'Amico, E., Segatore, B., de Massis, M. R., Bianchi, C., Luzzaro, F., Rossolini, G. M., Toniolo, A., Nicoletti, G. \& Amicosante, G. (2002). Molecular characterization of extended-spectrum $\beta$-lactamases produced by nosocomial isolates of Enterobacteriaceae from an Italian nationwide survey. J Clin Microbiol 40, 611-614.

Pitout, J. D. \& Laupland, K. B. (2008). Extended-spectrum $\beta$-lactamaseproducing Enterobacteriaceae: an emerging public-health concern. Lancet Infect Dis 8, 159-166.

Rodrigues, C., Shukla, U., Jog, S. \& Mehta, A. (2005). Extendedspectrum $\beta$-lactamase-producing flora in healthy persons. Emerg Infect Dis 11, 981-982.

Rodríguez-Baño, J., Navarro, M. D., Romero, L., Martínez-Martínez, L., Muniain, M. A., Perea, E. J., Pérez-Cano, R. \& Pascual, A. (2004). Epidemiology and clinical features of infections caused by extended-spectrum $\beta$-lactamase-producing Escherichia coli in non-hospitalized patients. J Clin Microbiol 42, 1089-1094.

Sahly, H., Navon-Venezia, S., Roesler, L., Hay, A., Carmeli, Y., Podschun, R., Hennequin, C., Forestier, C. \& Ofek, I. (2008). Extended-spectrum $\beta$-lactamase production is associated with an increase in cell invasion and expression of fimbrial adhesins in Klebsiella pneumoniae. Antimicrob Agents Chemother 52, 3029-3034.

Struelens, M. J. (1996). Consensus guidelines for appropriate use and evaluation of microbial epidemiologic typing systems. Clin Microbiol Infect 2, 2-11.

Talbot, G. H., Bradley, J., Edwards, J. E., Jr, Gilbert, D., Scheld, M. \& Bartlett, J. G. (2006). Bad bugs need drugs: an update on the development pipeline from the Antimicrobial Availability Task Force of the Infectious Diseases Society of America. Clin Infect Dis 42, 657668.

Tenover, F. C., Arbeit, R. D., Goering, R. V., Mickelsen, A., Murray, B. E., Persing, D. H. \& Swaminathan, B. (1995). Interpreting chromosomal DNA restriction patterns produced by pulsed-field gel electrophoresis: criteria for bacterial isolate typing. J Clin Microbiol 33, 2233-2239.

Thambu, D., Pichamuthu, K., Varghese, G. M. \& Subramanian, S. (2006). Community-acquired, fatal extended spectrum $\beta$ lactamase producing Klebsiella pneumoniae splenic abscess and sepsis. J Postgrad Med 52, 328-329.

The India Antimicrobial Resistance Study Group, Mathai, D., Rhomberg, P. R., Biedenbach, D. J. \& Jones, R. N. (2002). Evaluation of the in vitro activity of six broad-spectrum $\beta$-lactam antimicrobial agents tested against recent clinical isolates from India: a survey of ten medical center laboratories. Diagn Microbiol Infect Dis 44, 367-377.

Valverde, A., Coque, T. M., Sánchez-Moreno, M. P., Rollán, A., Baquero, F. \& Cantón, R. (2004). Dramatic increase in prevalence of fecal carriage of extended-spectrum $\beta$-lactamase-producing Enterobacteriaceae during non-outbreak situations in Spain. J Clin Microbiol 42, 4769-4775.

Weber, M. W., Carlin, J. B., Gatchalian, S., Lehmann, D., Muhe, L., Mulholland, E. K. \& WHO Young Infants Study Group (2003). Predictors of neonatal sepsis in developing countries. Pediatr Infect Dis J 22, 711-717. 